\title{
RISK AS A FACTOR IN DECISION-MAKING
}

\author{
Gueorgui STANKOV
}

\begin{abstract}
Incorporating the problems of risk in the management of an organization is an important issue for any manager, especially for those in the security sector. This article examines different approaches that enable taking into account the associated risks in decision-making. Considering a comprehensive understanding of risk, the transformation problems resolved in an organization and the types of factors in decision-making, three different approaches are described. Based on the Analytic Hierarchy Process (AHP) methodology, an integral criterion for selection of an alternative could be developed.
\end{abstract}

Keywords: Risk, risk management, multi-criteria decision-making, AHP.

Many of the decisions, including the strategic ones, made by people or organizations are complex and the expected outcomes are uncertain. This uncertainty stems from the limited possibility to take into account all influencing factors for these decisions or, in other words, the stochastic nature of these factors. This often leads to situations where the real outcome is different from the desired one. If the resources used to realize a decision are significant, then the deviations could cost a lot. This makes risk management an important process in any endeavor involving a great deal of resources.

Obviously, managing risk in itself will rarely be an objective. The objectives will often be formulated as "getting the best out of the resources at our disposal." This implies that on the way to achieve an objective, all decisions have to consider the associated risk (the level of uncertainty). In other words, the risk is a factor in decisions, which do not aim at the risk itself. The question is: "How can risk be taken in consideration in decision-making?"

The answer to this question will depend on our understanding of risk, the complexity of the decision to be made, the level of formalization, the management practice/ culture of the organization/ person, etc.

In this paper, the author attempts to analyze some quantitative approaches for considering risk in decision-making. To this end, the article begins with clarification of the 
term "risk." This is followed by a description of the main approaches to the problem considered. Conclusions about the applicability of the approaches are given afterwards.

\section{Definitions and Characteristics of Risk}

Many definitions of the term "risk" exist. Some are given below:

"Uncertainty inherent in plans and the possibility of something happening (i.e. a contingency) that can affect the prospects of achieving business or project goals."

"A measure of the potential inability to achieve overall program objectives within defined cost, schedule, and technical constraints and has two components: (1) the probability/ likelihood of failing to achieve a particular outcome, and (2) the consequences/ impacts of failing to achieve that outcome."

These definitions regard risk as something negative, i.e. the risk threatens the completion of the objectives. A fresher view on the understanding of risk can be found in the following definitions:

"A measure of future uncertainties in achieving program performance goals and objectives within defined cost, schedule and performance constraints."

"An uncertain event or condition that, if it occurs, has a positive or negative effect on a project's objectives."

This new look at risk brings some significant advantages:

- Risks are not only threats, but opportunities as well. Therefore, the group of events considered as risk is broader.

- A unified approach to the management (decision-making) of both opportunities and threats could be applied.

Any decision that will consider risk as a factor will depend on the characteristics of risk, which are derived from the definitions provided above:

- Risk means events that are not certain. It is a proactive and probabilistic category.

- Risk is a function of two variables: the probability of deviation from a particular outcome and the impacts of that deviation.

- There are two types of risk with regard to the consequences for achieving the decision objectives: negative and positive.

- Time is factor. Both probability and impacts usually depend on time.

- Risk is a subjective category. The assessment of risk depends on:

○ The entity which will be bearing the effects, i.e. the viewpoint. 


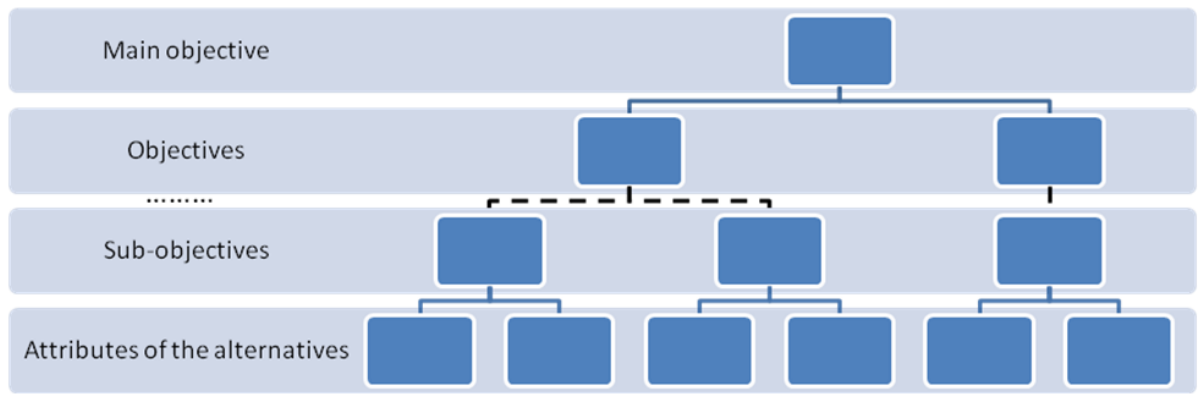

Figure 1: General Decision-Making Problem.

- The measurement of likelihood and impact, which are often subjective as well.

These characteristics may lead to adoption of different approaches when considering risk in decision-making.

\section{General Decision-Making Problem}

Most of the strategic decisions made by organizations or individuals are multi-criteria, i.e. they can be represented as choice of the most suitable development alternative, dictated by a system of goals. It is important to outline the following characteristics of the structure of the goals/objectives:

1. The structure of the goals/objectives/criteria is hierarchical, i.e. the objectives may have sub-objectives at several levels (see Figure 1).

2. The different goals/objectives/criteria have different weights, i.e. they have different importance.

The choice itself requires comparison of the worth (value) of the attributes of the alternatives with respect to the objectives. Combining the factors (attributes, objectives, sub-objectives, etc.) is required in order to make a decision. That means definition of:

- A common measure for the factors/attributes (incl. both quantitative and qualitative) at the lowest level.

- A common scale for the factors.

- The relative importance (weight) of each factor.

- The relationships between the factors, i.e. how do they relate to each other and to the utility function of the parent factor. 


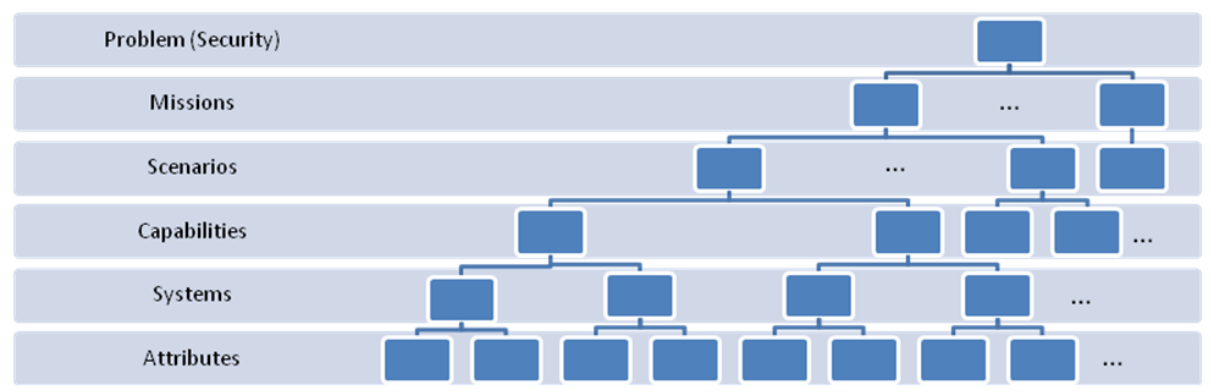

Figure 2: Defense Transformation Problem (An Example).

Developing a common measure of the attributes and a common scale means a proper definition of a utility function, representing how useful is the value of an attribute for the completion of an objective:

$$
\begin{aligned}
& u=f(x) \text { - utilityfunction } \\
& x \text { - attribute } \\
& 0 \leq u \leq 1
\end{aligned}
$$

If the utility of each attribute and the relationships between the factors in the hierarchy are known, then the utility (value) of each alternative could be calculated. For the weights of the factors of a level the following must be true:

$$
\sum_{i} w_{i}=1
$$

Decision-making in military organizations is not exception of this rule. Force transformation decisions are issues of choosing the best alternative with respect to a hierarchical set of defense goals/objectives. The different options are estimated according their "utility" for reaching the respective goals (see Figure 2).

A more formal approach to finding solutions to such problems requires the following types of techniques:

- For creation of the structure of the factors for decision-making (goals, objectives, criteria, etc.).

- For evaluation of the relative importance/weight of each goal/objective or criterion. Those weights should be consistent.

- For evaluation of the relative utility of the characteristics (attributes) of the different alternatives for achieving the goals at different levels. 
The Analytical Hierarchy Process (AHP) ${ }^{5}$ could be used as a model for solving this type of multi-criteria problems. It is an overall framework for selecting the option among the alternatives according to the developed hierarchical structure of goals/objectives/criteria/requirements. The AHP meets the requirements given above and a detailed description of the technique is given by Saaty. ${ }^{6}$ The best alternative (option) is the one that best meets the objectives.

\section{Types of Factors in Decision-Making}

In order to find an appropriate way to take into account risk in decision-making, it is necessary to understand how the different factors influence the decision in an AHP model. The factors can be categorized into several groups according to their role in the decision-making process:

- Features/attributes (of the alternatives). These factors are quantitative or qualitative characteristics of the alternatives that are considered in the decision.

- Objectives. These factors are measurable/calculable indicators for comparison of the alternatives. Usually they are given as a hierarchical structure of criteria for assessment.

- Utility of the attributes in regard to the objectives. Each attribute has different nature and therefore - different measure. The utility function creates a common measure for the attributes (eliminates the necessity to deal with different measures or dimensions) and creates a common scale for them (see formulae 1). The utility functions might have:

- A positive slope - higher value of the attribute means higher utility.

$$
\frac{d u}{d x}>0
$$

- A negative slope - higher value of the attribute means lower utility.

$$
\frac{d u}{d x}<0
$$

- Constraints. These factors represent limitations that exist with respect to this decision. Very often these limitations are for resources (time, money, workforce), but could be policy-related as well, e.g. defined in regard to quality, scope, risk and other matters, which the organization may impose on decision-making. The constraints could be fixed or variable. They define levels of acceptability of attributes or objectives (i.e. utility). 
- Weights. These factors represent the relative importance of the objectives or attributes to the decision-maker. They are used to define a way for calculation of the value of the objective (utility) at the next level of the hierarchical set of objectives.

The relationships between the factors of a certain level define the way to estimate the utility of the parent objective. According to their influence on the other factors of the same level (respectively, according to the way of calculation of the utility of the parent level), the factors could be subdivided into:

- Additive - the utility of such a factor (attribute or objective) is added to the utility of the rest; the utility of the parent objective is calculated using the following formulae:

$$
\begin{gathered}
U=\sum_{i=1}^{n} u_{i} w_{i} \\
\sum_{i=1}^{n} w_{i}=1
\end{gathered}
$$

- Multiplicative - the utility of such a factor (attribute or objective) is multiplied by the utility of the rest to obtain the utility of the parent objective:

$$
\begin{gathered}
U=\prod_{i=1}^{n} u_{i} w_{i} \\
\sum_{i=1}^{n} w_{i}=1
\end{gathered}
$$

Formulae 5 should be used for factors that are relatively independent, while formulae 6 - for cases where dependency of the factors is present.

\section{Approaches to Risk Decision-Making}

How could risk management be incorporated into the overall management of the organization? The answer to this question depends on the understanding what type of decision factor the risk is.

\section{Risk as a Constraint}

If we consider the risk as a constraint, we will treat it in a way similar to the other constrains in the decision (e.g. cost, time, utility, etc.). 
First, thresholds for acceptability have to be set for any factor in the decision to be made. They could look like:

$$
\begin{aligned}
& C \leq C_{\max } \text { - cost contstraint } \\
& T \leq T_{\max } \text { - timecontstraint } \\
& U \geq U_{\min } \text { - utilitycontstraint } \\
& R^{-} \leq R_{\max } \text { - negative risk contstraint } \\
& R^{+} \geq R_{\min } \text { - positiverisk contstraint }
\end{aligned}
$$

The risk constraints could be part of a risk policy within the organization or they could be especially identified for the case. They could be set for an attribute, objective or the entire case (decision) independently.

Second, the risk could be considered as a resource. In this approach, we are trying to maximize the value (benefits) per unit of the resource. Similarly, applying the approach to risk will result in the following decision criteria:

$$
\left\{\frac{\max \cdot \frac{U}{R}}{\min \cdot \frac{R}{U}}\right\} \quad \text { where } U \text { is utility, } R \text { - measure of risk }
$$

It should be mentioned that it does not matter whether we consider positive or negative risk, although the approach is usually applied to negative risk. The objective is to maximize/minimize the respective ratio for the case (decision). The utility for each option is calculated separately from the risk. Usually, there is no single decision criterion. Selection is made considering a set of criteria like the one in formulae 8 , where this time $R$ is any other resource (e.g. cost, time, etc.) relevant to the case.

\section{The Risk as an Attribute or Objective}

Another way to take risk into account in decision-making is to consider it as an attribute of a decision alternative. In this case, a utility function of the risk (as an attribute) has to be defined. Based on the definitions of risk given above, the utility of risk will be a function of two variables: impact, usually measured in cost units, and probability, i.e.

$$
u_{\text {Risk }}=f(C, P)
$$

If we generalize the approach, we may reconsider our understanding of constraints in 


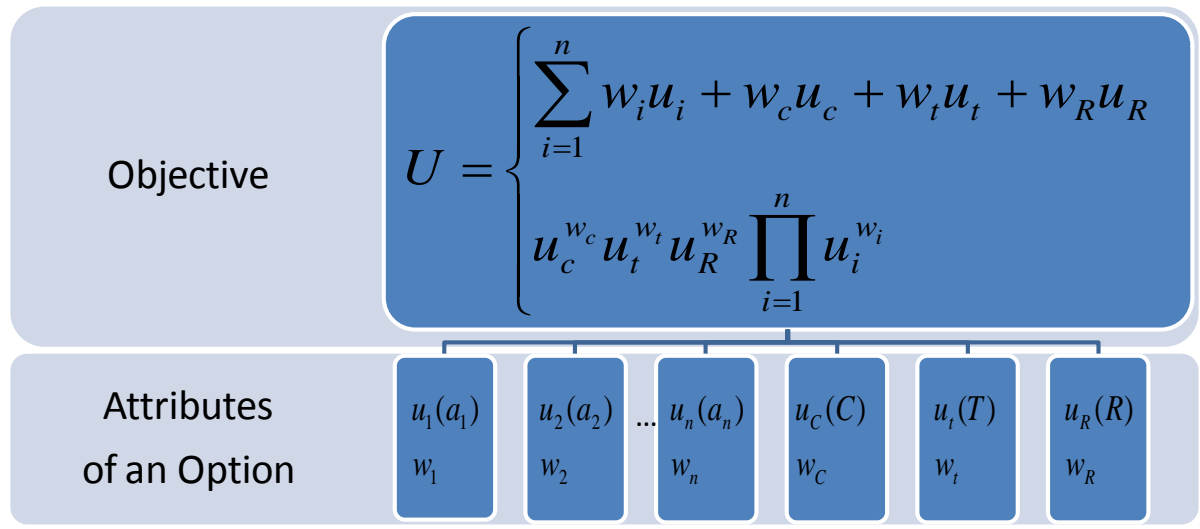

Figure 3: Calculation of Utility of an Objective at the First Level.

decision-making. If we assume variable constraints, we may treat them as attributes aswell. In this case, for any constraint (e.g. cost, time, etc.) we will have to define a utility function. This approach brings significant advantages:

- There is a single criterion for selection of the best alternative. Since all the constraints are built in this model, the only criterion for selection of the best alternative is its value (utility).

- $\quad$ Risk will be clearly defined throughout the hierarchical set of objectives. It can be assessed at any level for any objective.

Therefore, in order to implement this approach there are two issues that have to be resolved for any objective at any level:

- Calculation of utility;

- Calculation of risk.

The first issue is resolved using the AHP methodology. An example for calculation of the utility of an objective at the first level is given in Figure 3.

In this example, $a_{1} \div a_{n}$ are the attributes of an option, $C$ is cost, $T$ is time, and $R$ is a measure of risk. The calculation of utility of objectives at higher levels is trivial.

The second issue concerns the relationship between the risk of an objective and the risks of the respective sub-objectives. It has to be noted that this is optional for decision-making using this approach, but it provides some additional opportunities for analysis of the alternatives. The approach implies that for each objective the associ- 


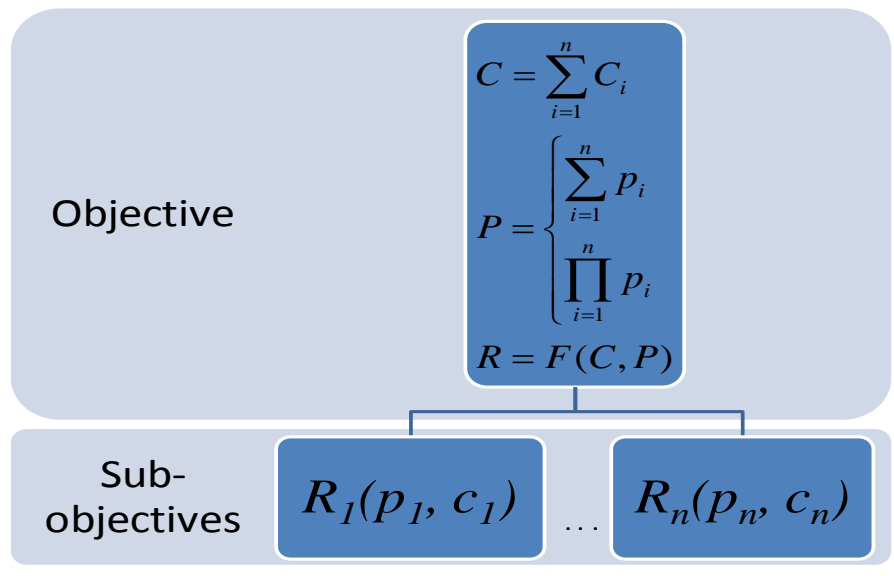

Figure 4: Relationship between the Risk of an Objective and the Risks of the Respective Subobjectives.

ated risk will be calculated. These risk calculations, for example, could be used to make utility-risk ratios for some specific analyses if necessary. Figure 4 presents the relationship between risks at different levels of decision objectives. There is a link between the components of risk (impact/cost and probability) at adjacent levels of the decision hierarchy. The cost of the objective will be sum of the costs of the sub-objectives. Similarly, there will be a probabilistic link between the decision levels. The risk function $R$ could be of different type. Its definition is out of the scope of this paper. It might be different depending on the nature of the decision to be made and the adopted understanding of the term "risk."

\section{How to Choose the Appropriate Method}

We have identified three approaches for considering risk as a factor in decisionmaking:

- Setting thresholds of risk acceptability;

- Using utility-risk ratios;

- Using risk utility functions.

Although all of the approaches are applicable to any situation or problem, the decision-maker might have a preference to one or another in different cases. The first approach could be used to discard alternatives that are too risky and therefore they could only aggravate further analysis of the alternatives. It might be a policy requirement in the organization and in some cases it might not be avoidable. 
The second approach is preferable when the decision concerns clearly defined results and maximizing the utility-risk ratio is important. And similarly to the first one, the risk is still considered separately from the formulated objectives. For rather complicated hierarchical sets of objectives, a large number of ratios might not be too helpful for the decision.

The third approach implies an integral (unified) selection criterion. For all decision factors, including risk, the respective utility is calculated. This fact facilitates the selection and provides opportunity to combine the approach with the other two approaches if necessary. This makes the third approach universally applicable to any case considered.

\section{Acknowledgement}

This paper reflects research on project SfP 981149 "Operations Research Support to Force and Operations Planning in the New Security Environment," sponsored by NATO's Scientific Affairs Division under the Science for Peace Program.

\section{Notes:}

1 Project Management - Part 3: Guide to the Management of Business Related Project Risk, BS 6079-3:2000 (British Standards Institution, 2000).

2 Risk Management Guide for DoD Acquisition, $5^{\text {th }}$ edition (version 2) (DoD, June 2003).

3 Risk Management Guide for DoD Acquisition, $6^{\text {th }}$ edition (version 1), (DoD, August 2006).

${ }^{4}$ A Guide to the Project Management Body of Knowledge (PMBOK ${ }^{\circledR}$ Guide) $3^{\text {rd }}$ edition (Project Management Institute, 2004).

5 Thomas L. Saaty, Multicriteria Decision Making: The Analytic Hierarchy Process (RWS Publications, 1990).

6 Saaty, Multicriteria Decision Making: The Analytic Hierarchy Process.

LTC GUEORGUI STANKOV - Information about the author is available on page 140 of this volume. 